\title{
Additional evidence for the genomic imprinting model of sex determination in the haplodiploid wasp Nasonia vitripennis: isolation of biparental diploid males after X-ray mutagenesis
}

\author{
C Trent, C Crosby and J Eavey \\ Department of Biology MS 9160, Western Washington University, Bellingham, WA 98226, USA
}

\begin{abstract}
The primary sex-determining signal in the haplodiploid wasp Nasonia vitripennis is not known. In haplodiploid reproduction, unfertilized eggs typically develop into uniparental haploid males and fertilized eggs into biparental diploid females. Although this reproductive strategy is common to all Hymenoptera, sex-determination is not strictly specified by the number of genome copies inherited. Furthermore, primary sex-determining signals differ among haplodiploid species. In the honeybee, for example, the primary signal is the genotype at a single, polymorphic locus: diploid animals that are homozygous develop into males while heterozygotes develop into females. Sex determination in Nasonia cannot be explained by this mechanism. Various lines of evidence show that the inheritance of a paternal genome is required for female sexual development and suggest a genomic imprinting mechanism involving an imprinted gene, ex-
\end{abstract}

pressed only from a paternal copy, that triggers female sexual development. In this model, haploid or diploid uniparental embryos develop into males due to a maternal imprint that silences this locus. The genomic imprinting model predicts that a loss-of-function mutation in the paternal copy of the imprinted gene would result in male sexual development in a biparental diploid embryo. In support of this model, we have identified rare biparental diploid males in the F1 progeny of X-ray mutagenized haploid males. Although uniparental diploid male progeny of virgin triploid females have been previously described, this is the first report of biparental diploid males in Nasonia. Our work provides a new, independent line of evidence for the genomic imprinting model of Nasonia sex determination.

Heredity (2006) 96, 368-376. doi:10.1038/sj.hdy.6800810; published online 22 March 2006

Keywords: haplodiploidy; sex determination; biparental males; genomic imprinting; Nasonia vitripennis; Hymenoptera

\section{Introduction}

The parasitic wasp Nasonia vitripennis has been the focus of genetic studies of hymenopteran developmental and evolutionary biology and recently was chosen for complete genome sequencing by the National Human Genome Research Institute (Page et al, 2002; Beukeboom and Desplan, 2003; Pultz and Leaf, 2003; Shuker et al, 2003; JH Werren, personal communication). As in other hymenopterans, Nasonia reproduces by haplodiploidy. This form of sexual reproduction typically produces uniparental males that develop from unfertilized eggs and inherit half of the maternal genome and biparental females that inherit half the maternal genome and the entire paternal genome. The ratio of male and female offspring of a mated wild-type female depends on the number of eggs that are fertilized. Despite the common theme of haplodiploidy, the primary sex-determining signal is known to vary among different hymenopteran species and has been clearly defined at the molecular level for only one: the honeybee Apis mellifera (Bull, 1983;

Correspondence: C Trent, Department of Biology MS 9160, Western Washington University, Bellingham, WA 98226-9160, USA.

E-mail: trent@biol.wwu.edu

Received 17 August 2005; accepted 15 January 2006; published online 22 March 2006
Cook, 1993; Beukeboom, 1995; Beye, 2004). In Apis, sex is determined by the genotype at a single, polymorphic locus called csd (complementary sex determiner), which has 19 known alleles (Adams et al, 1977). In this form of single-locus complementary sex determination, fertilized eggs that are heterozygous at the csd locus develop into females, fertilized eggs that are homozygous at this locus develop into biparental diploid (and usually sterile) males and unfertilized eggs develop into haploid, fertile males. The Apis csd gene has been cloned, but how the csd genotype triggers male or female development at the molecular level has not been elucidated (Beye et al, 2003).

The primary sex-determining signal in Nasonia is not known. Although complementary sex determination is thought to operate in at least 50 hymenopteran species (Cook and Crozier, 1995; Haig, 1998), sex determination in Nasonia vitripennis cannot be explained by this mechanism since highly inbred lines of Nasonia produce diploid females and haploid males (Skinner and Werren, 1980). Furthermore, observations made on polyploid strains of Nasonia indicate that, as in Apis and other haplodiploid species, ploidy per se is not the primary sexdetermining signal in this species (Table 1). Triploid females have arisen spontaneously in Nasonia cultures (Whiting, 1960). These females produce both haploid and diploid eggs. Unfertilized diploid eggs develop into fertile males. Although their average size is greater, 
Table 1 Female sexual development in Nasonia requires a paternal genome copy

\begin{tabular}{lll}
\hline Paternal gamete $^{\mathrm{a}}$ & \multicolumn{2}{c}{ Maternal gamete } \\
\cline { 2 - 3 } & $\begin{array}{l}\text { Haploid (from } 2 n \\
\text { or } 3 n \text { female) }\end{array}$ & $\begin{array}{l}\text { Diploid (from } 3 n \\
\text { female) }\end{array}$ \\
\hline None (unfertilized) & $\begin{array}{l}1 n \text { male } \\
1 m^{\mathrm{b}}\end{array}$ & $\begin{array}{l}2 n \text { male } \\
2 m\end{array}$ \\
Haploid (from 1n male) & $2 n$ female & $3 n$ female \\
& $1 m+1 p^{\mathrm{b}}$ & $2 m+1 p$ \\
Diploid (from $2 n$ male) & $3 n$ female & $4 n$ female \\
& $1 m+2 p$ & $2 m+2 p$ \\
Haploid $+\mathrm{PSR}^{\mathrm{c}}$ & $1 n$ PSR male & $2 n$ PSR male \\
& $1 m+\mathrm{pSR}$ & $2 m+\mathrm{PSR}$
\end{tabular}

${ }^{a}$ No reduction division in hymenopteran males.

${ }^{\mathrm{b}} m=$ maternal genome; $p=$ paternal genome.

${ }^{c} \mathrm{PSR}=$ paternal sex ratio chromosome.

diploid males are anatomically indistinguishable from haploid males. As there is no reduction division during meiosis in a hymenopteran male, diploid males produce diploid gametes and when mated to diploid females, produce triploid female progeny with one maternal and two paternal genome copies.

One triploid strain of Nasonia has been propagated for many generations and has been useful in examining various proposed models for sex determination in Nasonia (Whiting, 1960; Dobson and Tanouye, 1998; Beukeboom and Kamping, 2005). When a triploid female from this strain is mated with a haploid male, her diploid progeny are male if uniparental (developing from an unfertilized egg) and female if biparental (the product of a fertilization event). Triploid animals from this type of mating develop into females that carry two maternal and one paternal genome copies. The consistent theme emerging from these observations on Nasonia polyploids is that biparental offspring develop into females and uniparental offspring into males independent of their ploidy level. Two models for sex determination in Nasonia are consistent with this correlation between female sex determination and the presence of a paternal genome. In the fertilization model, the primary signal for female development is the fertilization event itself. In the genomic imprinting model proposed by Beukeboom (1995), female development is triggered in biparental animals by an imprinted gene that is expressed only from the paternal copy; the lack of expression of this gene results in male development in haploids and in diploid organisms containing only maternal genomes. Both of these models are consistent with observations on triploid strains, but the fertilization model is inconsistent with the results of experiments that uncouple the presence of a paternal genome copy from the fertilization of an egg.

Two mechanisms exist in Nasonia that result in loss of the paternal genome after a fertilization event: (i) cytoplasmic incompatibility induced in the zygote by the bacterium Wolbachia and (ii) the presence of the selfish paternal sex ratio (PSR) chromosome. In Nasonia, cytoplasmic incompatibility occurs when a male harboring the Wolbachia microorganism mates with an uninfected female (Werren, 1997; Tram and Sullivan, 2002).
PSR is a supernumerary chromosome carried by males that results in the loss of all paternal chromosomes (but itself) from a biparental embryo shortly after fertilization (Werren et al, 1987). In the presence of PSR or Wolbachiainduced cytoplasmic incompatibility, the paternal genome is lost and fertilized eggs develop as males, suggesting that fertilization per se is not sufficient to trigger female development in the absence of a paternal genome. In contrast, the genomic imprinting model is not contradicted by these data.

To confirm and extend these observations, Dobson and Tanouye (1998) used triploid Nasonia females fertilized by males carrying the PSR chromosome to examine the link between the paternal genome and sex determination in offspring of various ploidy levels. By showing that biparental triploid embryos carrying the PSR chromosome develop into diploid males after loss of the paternal genome, their work demonstrated an absolute correlation between female sex determination and the presence of a paternal genome and male sex determination and the absence of a paternal genome, independent of the ploidy of the embryo or whether it was generated by a fertilization event (Table 1).

Taken together, the experiments described above provide compelling evidence that a paternal genome copy is required for female sexual development. And, as with other model organisms whose sex-determining mechanisms have been analyzed in detail (such as Drosophila, Caenorhabditis elegans and Apis), it is likely that individual genes play key roles in sex-determination in Nasonia. In its simplest version, the genomic imprinting model implies the existence of an imprinted gene that triggers (or is at least required) for female sex determination and predicts that a loss-of-function mutation in the paternal copy would result in development of a biparental diploid male rather than a biparental diploid female.

Here, we use a mutagenesis approach to test this prediction. We describe the isolation and genetic analysis of biparental diploid male progeny of mutagenized male parents. All previously described Nasonia diploid males have been uniparental, carrying only maternal genomes (Whiting, 1960; Cook, 1993). As these experiments do not rely on the triploid strain used by other researchers or on the complete postzygotic destruction of the paternal genome, they supply an independent line of support for the genomic imprinting model of Nasonia sex determination, as well as direct evidence that female development in Nasonia vitripennis normally requires a gene that must be contributed via a male parent.

\section{Materials and methods}

\section{Culture of wasps on host pupae}

Nasonia (formerly known as Mormoniella) vitripennis is a parasitic wasp; the life cycle and culture of this organism has been described in detail by Whiting (1967) and Pultz and Leaf (2003). We used pupae of the blowfly Sarcophaga bullata as hosts; after eclosing, female wasps were fed $2 \%$ sucrose until they were given host pupae. Cultures of Nasonia were maintained at temperatures ranging from 18 to $28^{\circ} \mathrm{C}$. Virgin females were collected by breaking open the host (Sarcophaga) pupal case and collecting the female (Nasonia) pupae before they eclosed and mated with their brothers. 
Strains of Nasonia used in this study

Wild-type (designated as Comp + B1) and mutant strains of Nasonia vitripennis were obtained from Mary Anne Pultz (Western Washington University). The phenotypes and map locations of the mutant alleles used in this study have been previously described (Saul and Kayhart, 1956; Saul et al, 1965; Saul et al, 1967). The bl-13, pm-541, rdh-5 and st-5219 allele designations follow Saul et al (1967). The R-locus oyster and scarlet mutations were described by Whiting (1965) and are designated as $s t^{+}$oy (oyster) and $s t^{D R} o y^{+}$(scarlet) in this paper to indicate that they are tightly linked (no recombination has ever been observed between these mutant loci) yet complementary mutations. In the text, a Roman numeral listed after the allele indicates the linkage group assignment. Wild-type eye color in Nasonia is dark purple; scarlet mutations (st-5219 and $s t^{D R} o y^{+}$) result in a bright red eye; $r d h-5$ (reddish) eye color is dull red; and $s t^{+}$oy (oyster) animals have gray eyes. Scarlet, reddish and doubly mutant scarlet-reddish eye colors are easily distinguished; the oyster mutation is epistatic to other eye-color mutations.

X-ray mutagenesis: Adult males were collected $24-36 \mathrm{~h}$ after emergence and irradiated in 35X10 mm Petri dishes (10 males per dish) at doses ranging from 1000 to 6000R. The X-ray source was calibrated to deliver an output of $420 \mathrm{R} / \mathrm{min}$ operated at $3 \mathrm{~mA}$ and $70 \mathrm{kV}$. About $3 \mathrm{~h}$ after irradiation, the males were mated; after 18-24h the males were removed from the mating chambers and the females were given host pupae. In some cases, the males were remated with a new batch of females; in these second matings, the males were removed by $48 \mathrm{~h}$ postmutagenesis. The progeny of the mated females were collected and scored as they emerged from the host puparia. Males treated with doses higher than 3000R produced very few daughters. Most of the mutants listed in Table 2 were the progeny of males treated with 1000 or 2000R.

\section{Assaying brood viability}

Viability of progeny from diploid and triploid females was assayed as described by Pultz et al (2000).

\section{Results}

Isolation of biparental diploid males among the progeny of mutagenized haploid males mated with diploid females We devised an F1 genetic screen to identify mutations in a locus, designated as $S$, with genetic properties predicted by the genomic imprinting model: a loss-offunction mutation in the paternal copy would cause a diploid biparental male to develop from a fertilized egg (Figure 1). Diploid females, doubly or triply marked with recessive mutations, were mated with X-ray mutagenized haploid males and the F1 progeny were screened for rare biparental diploid males. Such males would be phenotypically wild type and easily distinguished from uniparental haploid males, which exhibit the recessive mutant phenotypes of the parental females. Male adults are easily and reliably distinguished from females by wing length and antennal color. The three males designated as Screen 1 diploid males were found among the F1 progeny of parental females homozygous for three unlinked, recessive mutations affecting body (pm-541 I) and eye color ( $r d h-5 I I$ and st-5219 III). A fourth male (referred to as the Screen 2 male) was isolated in the screen shown in Figure 1, which made use of eye-color mutations oyster $\left(s t^{+}\right.$oy) and scarlet $\left(s t^{D R} \quad o y^{+}\right)$on chromosome I as well as additional unlinked eye color (rdh-5 II) and body color (bl-13 III) mutations.

\section{Diploid males appeared only in the progeny of X-ray mutagenized males and at a frequency similar to that of mutations in marker genes}

The appearance of biparental males in our screens was dependent on parental mutagenesis. For Screens 1 and 2, parental males were treated with doses of $X$-rays ranging from 1000 to 3000R. Among the $21588 \mathrm{~F} 1$ progeny of these mutagenized fathers (Table 2), we identified four phenotypically wild-type males. In parallel experiments, we also examined over $18000 \mathrm{~F} 1$ progeny of unmutagenized males (Screen 3 in Table 2), but observed no biparental male progeny. We have also performed other F1 screens (using different genetic markers) with unmutagenized males. As in the Screen 3 controls, we found no evidence for spontaneous biparental male progeny in these experiments, which serve as additional controls and increase the number of unmutagenized genomes examined to over 28000 (data not shown).

The rate of appearance of the wild-type biparental males in our screens is consistent with that observed for other gene mutations. Throughout the screening process, new recessive mutations in the mutant eye-color loci carried by the parental females ( $r d h-5$ and st5219 in

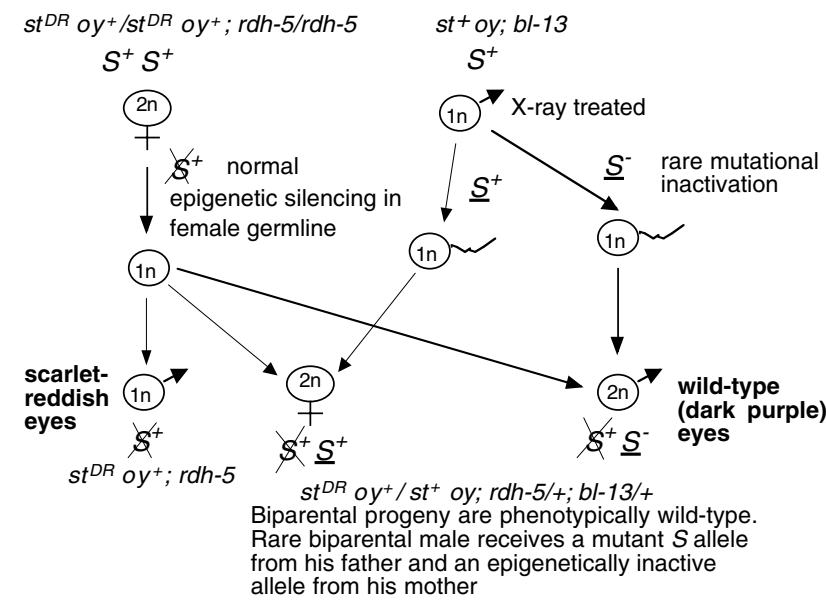

Figure 1 A genetic screen to identify the rare biparental diploid males predicted by the genomic imprinting model of sex determination. The $S$ allele indicates a hypothetical imprinted gene required for female sexual development that is expressed only if transmitted via the male germ line. An X over the allele indicates normal epigenetic silencing that would result from maternal transmission. The paternal $S$ allele is underlined. The parental markers used for Screens 1 and 2 are listed in Table 1; this figure shows genotypes for Screen 2. In both screens, rare biparental diploid males were identified by their wild-type eye color. In Screen 2, haploid $s t^{+}$oy; bl-13 males were mutagenized with $\mathrm{X}$-rays and mated to diploid females doubly marked with $s t^{D R} o y^{+}$and $r d h-5$. From such a mating, unfertilized eggs $\left(s t^{D R} o y^{+} ; r d h-5\right)$ will develop into haploid males with mutant scarlet-reddish eye color and fertilized eggs ( $s t^{D R}$ $o y^{+} / s t^{+}$oy; $\left.r d h-5 /+; b l-13 /+\right)$ will develop into phenotypically wild-type diploid females. 
Table 2 F1 screen for biparental diploid males

\begin{tabular}{|c|c|c|c|c|c|c|c|}
\hline Screen & $\begin{array}{l}\text { Genotype: } \\
\text { parental males }\end{array}$ & Genotype: parental females & $\begin{array}{l}\text { Paternal } \\
\text { genomes scored }\end{array}$ & $\begin{array}{l}\text { F1 progeny: } \\
2 N \text { males }\end{array}$ & $\begin{array}{l}\text { F1 progeny: } \\
\text { reddish females }\end{array}$ & $\begin{array}{l}\text { F1 progeny: } \\
\text { st-5219 females }\end{array}$ & $\begin{array}{l}\text { F1 progeny: } \\
s t^{D R} \text { females }\end{array}$ \\
\hline 1 & $\begin{array}{l}\text { X-ray treated } \\
\text { B1 (wild type) }\end{array}$ & pm-541; rdh-5; st-5219 & 7319 & $\begin{array}{l}2 \text { (ploidy } \\
\text { confirmed) } \\
1 \text { (died) }\end{array}$ & $\begin{array}{l}2 \text { new } r d h-5 \\
\text { mutations }\end{array}$ & $\begin{array}{l}3 \text { new st }-5219 \\
\text { mutations }\end{array}$ & $\mathrm{NA}^{\mathrm{a}}$ \\
\hline 2 & $\begin{array}{l}\text { X-ray treated } \\
s t^{+} \text {oy; bl-13 }\end{array}$ & $s t^{D R}$ oy ${ }^{+} ; \mathrm{rdh}-5$ & 14269 & $\begin{array}{l}1 \text { (ploidy } \\
\text { confirmed) }\end{array}$ & $\begin{array}{l}2 \text { new } r d h-5 \\
\text { mutations }\end{array}$ & NA & $\begin{array}{l}36 \text { new } s t^{D R} \\
\text { mutations }\end{array}$ \\
\hline \multicolumn{2}{|c|}{ Totals for Screen 1 and 2} & & 21588 & $\begin{array}{l}4 / 21588 \\
\sim 1 / 5400\end{array}$ & $\begin{array}{l}4 / 21588 \\
\sim 1 / 5400\end{array}$ & $\begin{array}{l}3 / 7319 \\
\sim 1 / 2400\end{array}$ & $\begin{array}{l}36 / 14269 \\
\sim 1 / 400\end{array}$ \\
\hline 3 Control & $\begin{array}{l}\text { Unmutagenized } \\
\text { B1 (wild type) or } \\
s t^{+} \text {oy; } b l-13\end{array}$ & $\begin{array}{l}\text { pm-541; rdh-5; st-5219 or } \\
\text { st }{ }^{D R} \text { oy } ; \text { rdh-5 }\end{array}$ & 18045 & 0 & 0 & 0 & 0 \\
\hline
\end{tabular}

${ }^{\mathrm{a}} \mathrm{NA}=$ not applicable.

Screen 1 and $s t^{D R}$ and $r d h-5$ in Screen 2), were tracked by examining the F1 females for mutant reddish or scarlet eye colors. (The wild-type Nasonia eye color is dark purple.) The data presented in Table 2 show that the frequency of F1 diploid males is very similar to the frequency of new mutant alleles at the $r d h-5$ and st5219 loci. The exceptionally high rate of X-ray-induced mutations at the $s t^{D R}$ locus has been previously reported (Whiting, 1956; Caspari, 1958) and may be due to an unusually large target size for this gene.

The exceptional wild-type males are biparental with one maternal and one paternal genotype

The phenotypically exceptional wild-type male progeny exhibited normal male anatomy with respect to wing length, antennae and external genitalia. These unusual males appeared to be biparental, carrying both a maternal and paternal genome. Owing to the absence of a reduction division, diploid Nasonia males produce diploid gametes. When mated to diploid females, they produce triploid daughters carrying a single maternal genome copy (from their diploid mother) and two paternal genome copies (Table 1 and Figure 2). Triploids, carrying two maternal genomes and one paternal genome, can also be produced by crossing triploid females with haploid males. These triploids also develop into females, showing that a single copy of the paternal genome is sufficient to trigger female development in a triploid embryo. We predicted that when mated to diploid females, the diploid males found in our screens would produce triploid female progeny since the single $S^{+}$allele carried by a $S^{+} S^{-}$diploid male should be sufficient to trigger female development in $S^{+} S^{-} S^{+}$progeny (where $\mathcal{S}^{+}$indicates the maternally inactive allele).

Three of the phenotypically wild-type males discovered in our screens exhibited normal mating behavior and fertility and produced daughters when mated with diploid females. (the fourth male, isolated in Screen 1, died before mating.) We used two different approaches to demonstrate that the daughters were triploid: (1) by showing they produced viable progeny only rarely and at the expected frequency and (2) by examining the segregation pattern of eye-color markers in their viable progeny (Figure 2). All triploid females described in these experiments are the first generation daughters of the biparental diploid males.

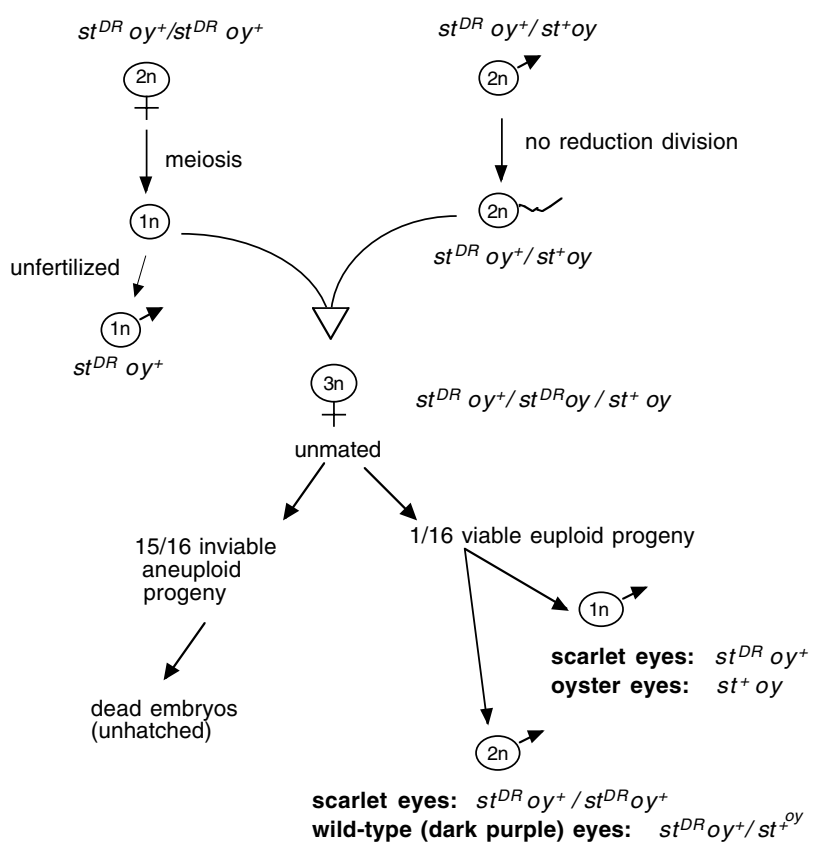

Figure 2 The progeny of the biparental diploid males are triploid females that produce haploid and diploid males. Diploid males produce diploid gametes and when mated to diploid females produce triploid female progeny. The $s t^{D R} o y^{+} / s t^{+}$oy; $r d h-5 /+$; $b l-13 /+$ Screen 2 male (Figure 1) was mated with $s t^{D R}$ oy ${ }^{+} ; r d h-5$ females to produce triploid daughters of genotype $s t^{D R} o y^{+} / s t^{D R}$ $o y^{+} / s t^{+}$oy; $r d h-5 / r d h-5 /+; b l-13 /+/+$. The genotypes and phenotypes indicated in this figure are for the R-locus only, which acts as a single segregation unit with no recombination between the $s t^{D R}$ and oy alleles. Unmated, the triploid daughters produced haploid scarlet $\left(s t^{D R} o y^{+}\right)$and oyster $\left(s t^{+} o y\right)$ male progeny and diploid scarlet $\left(s t^{D R}\right.$ $\left.o y^{+} / s t^{D R} o y^{+}\right)$and wild-type $\left(s t^{D R} o y^{+} / s t^{+} o y\right)$ male progeny. The unlinked eye-color mutation, $r d h-5$ (reddish), was also segregating in the progeny of these females. The R-locus genotypes can easily be assessed in either a $r d h-5^{+}$or $r d h-5$ background (see Materials and methods). The $b l-13$ mutation affects body color.

Triploid females are highly fecund but produce a limited number of viable progeny because most gametes are aneuploid, due to the random segregation during meiosis of the three homologs of each chromosome. Products of meiotic divisions with balanced chromosome sets produce viable haploid or diploid eggs. As $n=5$ in Nasonia vitripennis, $2\left(\frac{1}{2}^{5}\right)$ or $\frac{1}{16}(6.25 \%)$ of the gametes produced by a triploid female are expected to be 
balanced ( $1 n$ or $2 n)$ and produce viable progeny. As detailed in the Supplementary information, this predicted value agrees with the frequency of embryonic death observed for the progeny of 25 daughters of the Screen 2 diploid male $\left(n=530\right.$ embryos $\chi^{2}=1.069$; $P=0.3$ ). The daughters of the Screen 1 males also gave rise to broods with very high frequencies of embryonic lethality (data not shown).

The ploidy of the daughters of the diploid males was confirmed independently by examining the segregation of eye-color markers in the progeny of these animals. Here, we describe observations on the daughters of the Screen 2 male, which was heterozygous for two complementing 'alleles' of the $R$ locus (Linkage group I): $s t^{+}$oy (oyster) and $s t^{D R} \mathrm{oy}^{+}$(scarlet). Although the $s t^{D R}$ $o y^{+}$and the $s t^{+}$oy mutations complement to produce wild-type eye color in heterozygous animals, recombination between these mutations has never been observed: virgin diploid $s t^{D R} o y^{+} / s t^{+}$oy females produce $s t^{+}$oy and $s t^{D R} \mathrm{Oy}^{+}$males only. (Whiting, 1965, analyzed the progeny of over 25000 progeny of virgin $s t^{D R} \mathrm{oy}^{+} / s t^{+}$oy mothers.) This unusual genetic feature of the $R$-locus allowed us to distinguish between haploid and diploid males and between diploid and triploid females in the subsequent generations. In this and following sections and in the figures, the genotypes given include only those genetic markers relevant for the particular experiment under consideration. Complete genotypes are given in the figure legends. Genetic observations confirming that daughters of the Screen 1 males were triploid are described in the Supplementary information.

We generated triploid daughters of genotype $s t^{D R} \mathrm{oy}^{+} /$ $s t^{D R} o y^{+} / s t^{+}$oy by mating the Screen $2\left(s t^{D R} \mathrm{oy}^{+} / s t^{+}\right.$oy) male with $s t^{D R} o y^{+}$diploid females. Virgin triploid females of this genotype should produce males showing three R-locus eye-color phenotypes: haploid scarlet ( $s t^{D R}$ $\left.o y^{+}\right)$and oyster $\left(s t^{+}\right.$oy) male progeny and diploid scarlet $\left(s t^{D R} o y^{+} / s t^{D R} o y^{+}\right)$and wild-type $\left(s t^{D R} o y^{+} / s t^{+} o y\right)$ male progeny (Figure 2). We examined the eye color of the rare viable male progeny produced by these females and observed scarlet, oyster and wild-type males within single broods of many individuals. Furthermore, we confirmed that the phenotypically wild-type males were diploid: when mated to diploid females, they produced triploid female progeny.

These observations show unequivocally that the exceptional wild-type males found in our screens were biparental diploids, which, when mated to diploid females, generated triploid daughters carrying two paternal and one maternal genome copies. This is the first new report of diploid males and triploid females in Nasonia since Whiting (1960) described three independent, spontaneous events resulting in Nasonia polyploids. In contrast to the diploid males described here, the polyploids described by Whiting originated from the production of rare diploid gametes in the germ line of diploid females. For example, one event was detected as a uniparental diploid male identifiable by its R-locus genetic markers.

The sex-determining lesion is not a Mendelian dominant masculinizing allele

The biparental diploid males appeared among the first generation progeny of mutagenized males. Although the mutations in the diploid males transformed biparental embryos (which normally would have developed into females) into males, these mutations had no obvious phenotypic effect on the triploid progeny of these males. As shown in Figure 1, each of the diploid males may be heterozygous for a loss-of-function allele in an imprinted gene required for female sex determination. Alternatively, the sex-determining lesion could result from a ploidy-sensitive Mendelian dominant mutation (designated as $T$ in this section and in Figure 3) that causes transformation of a diploid $\left(T / t^{+}\right)$biparental animal into a phenotypic male without affecting a triploid $\left(T / t^{+} / t^{+}\right)$ animal. In this scenario, the sensitivity of the phenotype to the number of doses of the wild-type allele could be explained by a dominant, loss-of-function mutation in a haploinsufficient gene or by a dominant-negative mutation - the phenotype of either type of dominant allele would be expected to be sensitive to the number of wildtype alleles present in the organism (Muller, 1932; Wilkie, 1994).

Regardless of the specific mechanism of dominance, a diploid animal heterozygous for a Mendelian dominant allele should exhibit the dominant phenotype independently of whether the allele was inherited from its father or its mother. If the triploid daughters of the diploid males are carrying a Mendelian dominant (ie, are $T / t^{+} /$ $t^{+}$), when mated to haploid $\left(t^{+}\right)$males their progeny should include a class of biparental diploid $\left(T / t^{+}\right)$males (Figure 3). (As shown in Table 1, biparental diploid males

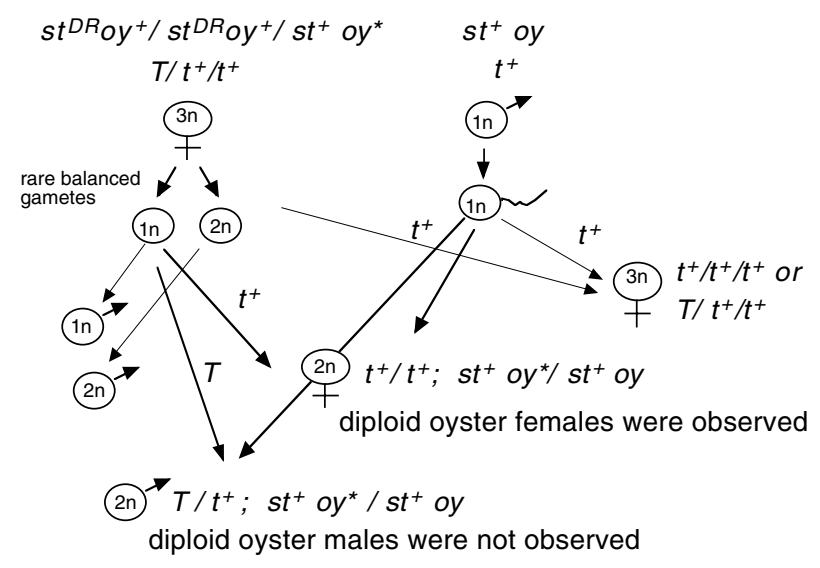

A ploidy-sensitive Mendelian dominant mutation should result in biparental males that would be recognized as diploid oyster males. Not all genotypic classes are shown in this figure.

Figure 3 Biparental diploid males are not observed in the progeny of mated triploid daughters. The phenotypic effect of a ploidysensitive Mendelian dominant mutation $(T)$ would be exhibited in a biparental diploid regardless of whether the allele was transmitted via the male or female parent: diploid heterozygotes receiving the $T$ allele from their triploid mother and a wild-type allele from their father will develop into biparental males. (In contrast, as shown in Figure 4, a mutation in an imprinted gene will show a paternal effect.) Triploid $s t^{D R} o y^{+} / s t^{D R} o y^{+} / s t^{+}$oy; $r d h-5 / r d h-5 /+; b l-13 /+/+$ daughters of the Screen 2 diploid male were mated with $s t^{+}$oy; $b l$ 13 males and their broods examined for the presence of $T / t^{+}$oyster biparental diploid males. No such biparental males were observed indicating that the sex-determining mutation in this diploid male was not a Mendelian dominant. The $s t^{+} o y^{*}$ allele was carried by the mutagenized males in Figure 1. In this figure, we assume that the sex-determining mutation $T$ and the R-locus are unlinked. The genotypes and phenotypes of uniparental males produced by the triploid females are shown in Figure 2. 
are not observed in the progeny of the previously established triploid line of Nasonia used by other investigators.)

The $s t^{D R} o y^{+} / s t^{D R} o y^{+} / s t^{+} o y^{*}$ genotype of the Screen 2 diploid male's triploid daughters allowed us to determine whether, when mated to haploid $s t^{+}$oy males, these females produced biparental male progeny. (The asterisk indicates the allele carried by the mutagenized grandparent.) As shown in Figure 3, the biparental progeny of a $T / t^{+} / t^{+}$animal would consist of diploid (T/ $\left.t^{+}\right)$males, diploid $\left(t^{+} / t^{+}\right)$females and triploid $\left(t^{+} / t^{+} / t^{+}\right.$ and $\left.T / t^{+} / t^{+}\right)$females. The biparental diploid progeny would have either wild-type $\left(s t^{D R} o y^{+} / s t^{+} o y\right)$ or oyster $\left(s t^{+} o y^{*} / s t^{+}\right.$oy) eyes.

Our analysis focused specifically on progeny with oyster eye color since diploid oyster males would necessarily be biparental, whereas diploid males with wild-type eye color could be either uniparental (Figure 2) or biparental. The mated triploid females collectively produced 48 oyster (diploid) females and 19 oyster males. All of the oyster males were haploid (uniparental): when mated to a diploid female, each male produced diploid daughters, as measured by fecundity. Assuming independent assortment between the R-locus and the sexdetermining mutation, when mated to $t^{+}$males, the $T / t^{+} / t^{+}$females should produce one diploid oyster male $\left(T t^{+}\right)$for every two diploid oyster females $\left(t^{+} t^{+}\right)$. The absence of diploid oyster males among the 48 diploid oyster animals produced in these crosses is statistically significant $\left(\chi^{2}=24, \mathrm{df}=1 ; P=<0.0001\right)$ and clearly inconsistent with the genetic behavior predicted for a Mendelian dominant mutation. If the oyster $\left(s t^{+} o y^{*}\right)$ allele from mutagenized male parent and the sexdetermining mutation were linked, the expected ratio of diploid oyster males:females would be even greater than the 1:2 ratio used in this analysis and would increase as the linkage distance decreases, to a maximum of $100 \%$ oyster males.

The data presented in this section are inconsistent with a ploidy-sensitive dominant masculinizing mutation $(T)$ since mated females carrying this mutation failed to produce biparental male progeny. However, as described in the next section, these observations are consistent with the genetic behavior predicted for an imprinted gene required for female sexual development and expressed only from a paternal copy.

\section{Is the sex-determining lesion transmitted via haploid males?}

A mutation in an imprinted gene $(S)$ that is dependent on paternal transmission for expression should show a paternal effect in its transmission: heterozygotes receiving the mutant allele via the mother will show no phenotypic effect (Figure 4a). In contrast, assuming that haploid males with the mutant allele are viable, paternal transmission of the mutation will result in a mutant phenotype in heterozygous offspring (Figure 4b). Consistent with a paternal effect, no phenotypic effect of the mutant allele (carried by the Screen 2 diploid male) could be detected in the biparental diploid progeny of mated triploid daughters (Figures 3 and 4a). However, if haploid $S^{-}$male progeny of $S^{+} / S^{+} / S^{-}$females are mated with wild-type diploid $S^{+} / S^{+}$females (Figure $4 \mathrm{~b}$ ), the $\mathcal{S}^{+} / S^{-}$heterozygous progeny should exhibit the mutant a

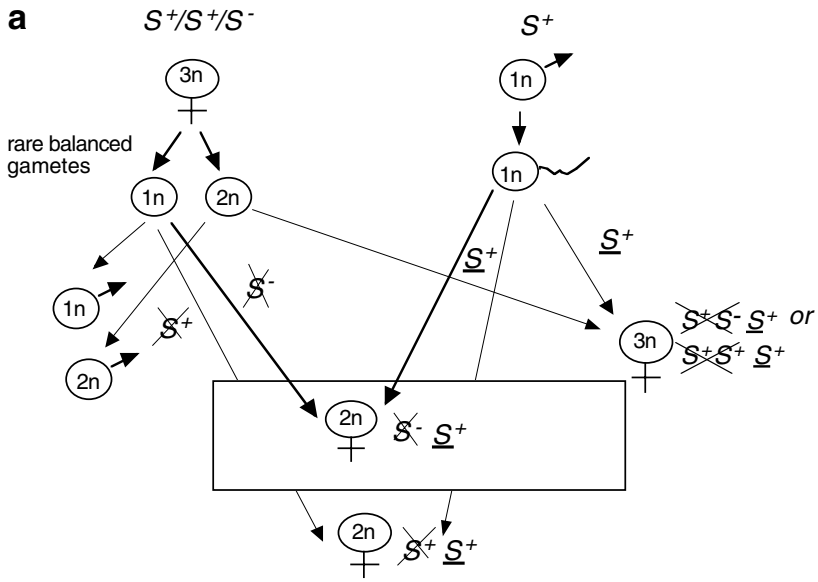

Biparental diploid animals receiving the mutant $S^{-}$allele from their mother will develop normally as females because of the $S^{+}$contribution of the male parent. Compare to $T / t^{+}$in Figure 3 .

b

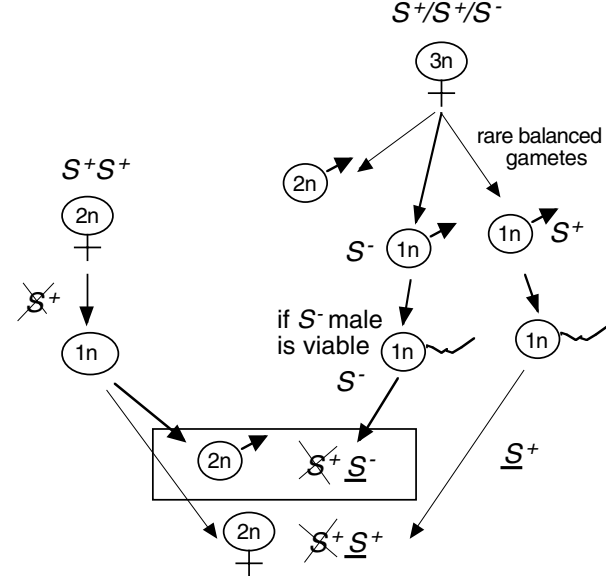

Biparental diploid animals receiving the mutant $S^{-}$allele from their father should develop into males rather than females.

Figure 4 The sex-determining mutations were not transmitted via haploid males. A mutant allele in an imprinted gene $(S)$ that is expressed only from the paternal copy will show a paternal effect pattern of transmission: biparental heterozygotes who inherited the allele from their mother will develop normally as females (a), whereas biparental heterozygotes who inherit the allele from their father should develop as males (b). An X over the $S$ allele indicates that it is not expressed in the developing embryo due to a maternal imprint that silences the allele; the paternal $S$ allele is underlined. (a) Transmission of the $S^{-}$allele via a female parent will not result in male development in biparental diploid heterozygotes because of the contribution of the $S^{+}$allele from the wild-type male parent. As described in the legend to Figure 3, we observed no biparental diploid males in the progeny of mated triploid daughters of the Screen 2 diploid male. (b) In contrast, transmission of the $S^{-}$allele via haploid males should result in male development in biparental diploid heterozygotes. Haploid male progeny were collected and mated with appropriately marked diploid females and their progeny examined for the presence of biparental diploid males. For example, haploid $s t^{+} o y^{*}$ males (identified by their oyster eyes) segregating from $s t^{D R} o y^{+}\left|s t^{D R} o y^{+}\right| s t^{+} o y^{*} ; r d h-5 / r d h-5 \mid+^{*} ; b l-13^{*}$ $+1+$ females were mated with $s t^{D R} o y^{+}$diploid females and the cross progeny scored for phenotypically wild-type $s t^{D R} \mathrm{oy}{ }^{+} / s t^{+} o y^{*}$ biparental males. No such males were observed in any of the crosses performed, suggesting that the mutant allele is lethal in haploids. Alleles marked with an asterisk were carried by the mutagenized males in Figure 1. 
phenotype: biparental diploid embryos will develop into males rather than females.

The triploid daughters of the diploid males carry the sex-determining mutation and two wild-type copies of the locus, one contributed from each parent (Figure 4). For each of the three biparental males isolated in our screens, at least 25 haploid male grandsons were mated with diploid females that were genetically marked so that biparental and uniparental male progeny could be differentiated. In all matings, only biparental female and uniparental male progeny were obtained. For example, an oyster male segregating from a Screen $2 s t^{D R} O y^{+} / s t^{D R}$ $o y^{+} / s t^{+} o y^{*}$ triploid female must be haploid. Assuming that the sex-determining mutation is unlinked to the $s t^{+}$ $o y^{*}$ allele (which was carried by the mutagenized haploid great grandfather), that there is no segregation bias and no loss of viability of haploid males carrying the mutation, then each oyster male has a $\frac{1}{3}$ probability of carrying the mutant sex-determining allele. Testing 25 individual oyster males corresponds to a probability $>99.99 \%\left(1-\frac{2}{3}^{25}\right)$ of mating at least one male with the sex-determining mutation. Additional details on these experiments (for both Screens 1 and 2 progeny) are included in the Supplementary information.

In summary, despite extensive testing we were unable to transmit a sex-determining mutation via the haploid male grandprogeny of the three diploid males found in our screens. One possible explanation is that the mutations are lethal in the haploid state and cannot be propagated via a haploid male (Figure 4b). Next, we demonstrate the presence of recessive lethal mutations in the triploid daughters of the diploid males.

\section{Presence of a zygotic lethal gene mutation in the diploid granddaughters of the diploid males}

Mated triploid females should produce both diploid and triploid female progeny (Table1 and Figure 3). Interestingly, for each of the three diploid males, there were three classes of granddaughters: clearly triploid $(<10 \%$ of progeny embryos viable); clearly diploid ( $>80 \%$ of progeny embryos viable); and a class of females that produced approximately 40-60\% dead embryos (data not shown). This latter class appeared to represent diploid females heterozygous for a zygotic mutation resulting in embryonic lethality.

For granddaughters of the Screen 2 male, we used R-locus eye-color phenotype to independently confirm ploidy. This allowed us to differentiate unambigously between embryonic death resulting from triploid aneuploidy and embryonic death due to heterozygosity for a lethal gene mutation and to establish a diploid female line that carried a recessive zygotic lethal mutation. This lethal mutation exhibits a Mendelian segregation pattern: virgin heterozygous females produce an equal number of viable and inviable haploid embryos (data not shown). No evidence was seen for linkage of the embryonic lethal mutation to either the $s t^{+}$oy (I) or $r d h^{+}$(II) or $b l-13$ (III) alleles, all of which were carried by the mutagenized grandfather (data not shown).

\section{Discussion/Conclusions}

The genomic imprinting model of Nasonia sex determination proposes the existence of an imprinted gene required for female sex determination and predicts that the absence of a paternally imprinted copy in the developing embryo, either normally in the progeny of virgin mother or abnormally due to a loss-of-function mutation, would result in male development. In this paper, we describe the isolation and analysis of three biparental diploid males in the F1 progeny of X-ray mutagenized fathers. Although uniparental diploid males are routinely observed in the progeny of unmated triploid Nasonia females, biparental males carrying both a maternal and paternal genome have not been previously described.

The appearance of rare biparental diploid males in the F1 generation following mutagenesis of the father is consistent with either a loss-of-function mutation in an imprinted gene or with a ploidy-sensitive Mendelian dominant allele that transforms biparental heterozygous diploids into males but has no effect on a triploid embryo carrying two wild type and one mutant copy of the gene. Mated triploid daughters of the Screen 2 male did not give rise to biparental diploid male progeny. This observation is inconsistent with a Mendelian dominant, which should transform heterozygous diploid embryos into biparental males irrespective of which parent contributed the mutant allele. However, this observation is consistent with the paternal effect predicted for a mutant allele of an imprinted gene that is normally expressed only from the paternal allele. The genetic markers used in Screen 1 did not allow us to exclude the possibility that the males generated in this screen were the result of a ploidy-sensitive dominant mutation, although it is likely that they resulted from the same type of mutation as the Screen 2 male.

To reveal a paternal effect pattern of transmission, a mutation disrupting female sex determination in Nasonia must be transmitted via haploid rather than diploid males because heterozygous diploid males do not have a meiotic reduction division and a single wild-type paternal allele in the heterozygous diploid is sufficient to trigger female sex determination in the triploid progeny. For each of the three diploid male isolates examined, we were unable to observe transmission of the mutation via haploid male progeny of the triploid daughters. This observation suggests that the mutations responsible for the biparental males are lethal in haploid animals. In diploid grand-daughters of the Screen 2 diploid male, a recessive zygotic embryonic lethal mutation was identified and strains carrying this allele were established. Examination of the diploid granddaughters of the Screen 1 biparental males also suggested the presence of a lethal allele, but strains carrying the mutations were not established. The relationship of these zygotic lethal gene mutations to the sex-determining mutations is tentative, as unrelated background mutations may well have been generated by the X-ray mutagenesis.

The exact nature of the lesions that generated the three biparental males discovered in our screens is not known. A loss-of-function mutation could affect the functioning of the product of an imprinted gene or could render the gene unable to be properly epigenetically modified. The lethality apparently associated with the mutations could represent a pleiotropic effect, indicating a role of the maternal gene copy in male (and presumably female) embryonic development as well as in female sexual development. This scenario would require expression of 
the gene from the maternal allele; the normal imprint could result in lower levels of expression from the maternal allele rather than complete inactivation or the imprints on the maternal and paternal alleles could confer different temporal or spatial expression of the alleles. For many imprinted loci in mammals, both maternal and paternal copies of the gene are known to be expressed in some tissues at some point during development (DeChiara et al, 1991; Spencer, 2002). Alternatively, the recessive lethality associated with the sex-determining lesion could result from a closely linked mutation in an unrelated gene. Since X-rays are known to induce chromosome mutations, it is possible that each of the three mutational events involved a deletion (or other rearrangement) that included the imprinted sex-determining locus and an adjacent gene or genes required for viability.

The experiments presented in this paper are consistent with previous observations demonstrating a requirement for a paternal genome in female sexual development. Using the triploid line originally described by Whiting (1960) and also used by Dobson and Tanouye (1998), Beukeboom and Kamping (2005) have recently described the rare appearance of uniparental females arising from unfertilized diploid eggs produced by unmutagenized triploid Nasonia females. These uniparental females may arise from rare imprinting failures in the triploid maternal germ line of a sex-determining gene that triggers female sex-determination but is normally expressed only from the paternal copy. The failure to silence such a gene by normal epigenetic modification would result in the activation of female sexual development in uniparental animals. Stochastic imprinting errors and individual variation in the level of expression of the 'silenced' allele have been described in mammals for various imprinted genes (Croteau et al, 2001; Sakatani et al, 2001; Bestor, 2003).

Beukeboom and Kamping (2005) found that, in contrast to the triploid females they examined, diploid Nasonia females do not produce rare uniparental female progeny. Various explanations for this difference include the possibility that a triploid dose of the imprinted sexdetermining locus results in a higher probability of a maternal imprinting failure. In other words, assuming that the default epigenetic state of the sex-determining gene renders it transcriptionally active and that silencing requires an active epigenetic modification, the extra genome copy may result in a higher level of imprinting noise, perhaps due to competition for rate-limiting factors involved in the epigenetic modification event.

Epigenetic lesions could also explain the observations described in this paper. The biparental diploid males discovered in our screens could have resulted from an imprinting defect in the X-irradiated paternal germ line, generating an epigenetic lesion rather than a loss-offunction mutation. This epigenetic lesion would have 'disappeared' in the next generation when imprints are erased (reset), presumably during germ cell development (Surani, 2001). In this scenario, there would be no sex-determining mutation per se and the zygotic lethal mutations observed in the descendants of the males would be incidental to the sex determination lesion. Although a stochastic epigenetic effect involving a transient imprinting mistake is possible, the data we have presented are best explained by a mutation in an imprinted gene. First, in contrast to the observations of Beukeboom, the appearance of biparental diploid males required mutagenesis of the male parents. We performed the same screens on the same scale with unmutagenized and X-ray mutagenized fathers and biparental diploid males were only observed in the progeny of mutagenized fathers. Second, the frequency of appearance of this mutant phenotype (one hit in about 5400 mutagenized paternal genomes) is similar to that observed for the appearance of new mutant alleles in the $r d h-5$ and st5219 genes used as maternal genetic markers in our screens. Third, our experiments did not involve polyploid parents which, as proposed above, might show increased noise with respect to imprinting events. Fourth, the examples referenced above of imprinting failures in mammals involve expression of the 'silenced' allele of the imprinted gene. To explain our observations, the imprinting defect in the biparental diploid males would necessarily be the opposite: the abnormal silencing of the 'active' allele.

The experiments presented in this paper support the genomic imprinting model for Nasonia sex determination by providing direct evidence for a paternally inherited gene that is required for sexual development in Nasonia females and is a candidate for the primary sexdetermining signal in this species. The existence of a heritable epigenetic mark that cues differential gene expression dependent on the parent of origin has been well documented for specific genes in mammals (for a review see: Reik and Walter, 2001). However, few examples of individual imprinted genes have been described for insects (Herrick and Seger, 1999; Lloyd, 2000). The Nasonia model system provides the opportunity to define such genes using classical genetic approaches and to study them at the molecular level using genome-era tools, which include a RAPD map based on interspecific crosses between $N$. vitripennis and a related species $N$. giraulti; high-density microsatellite and AFLP maps; BAC and EST libraries; and complete genome sequences of $N$. vitripennis, $N$. giraulti and a third Nasonia species, N. longicornis (Gadau et al, 1999; Pultz and Leaf, 2003; Ruetten et al, 2004; J Werren, personal communication). In addition, the molecular characterization of sex determination in the honeybee and the completion of its genome sequence will facilitate the exploration of the molecular basis of sex determination in Nasonia and comparative studies of sex determination in haplodiploid species that use different primary sexdetermining signals (Beye et al, 2003; Beye, 2004; Evans et al, 2004).

\section{Acknowledgements}

We thank Mary Anne Pultz for the initial inspiration to explore sex determination in a haplodiploid model organism. Throughout the project Mary Anne provided thoughtful input and enthusiastic support. We thank Leo Beukeboom for communicating his work on uniparental females before publication and Barbara Wakimoto for use of the X-ray facility at University of Washington. We also thank Leo Beukeboom, David Leaf and Mary Anne Pultz for providing comments on the manuscript and Tera Matson for assistance with wasp rearing and stock maintenance. This work was funded by National Institutes of Health Grant R15-GM57805-01. 


\section{References}

Adams J, Rothman ED, Kerr WE, Paulino ZL (1977). Estimation of the number of sex alleles and queen matings from diploid male frequencies in a population of Apis mellifera. Genetics 86: 583-596.

Bestor T (2003). Imprinting errors and developmental asymmetry. Philos Trans $R$ Soc Lond B Biol Sci 358: 1411-1415.

Beukeboom L, Desplan C (2003). Nasonia. Curr Biol 13: R860.

Beukeboom LW (1995). Sex determination in Hymenoptera: a need for genetic and molecular studies. BioEssays 17: 813-817.

Beukeboom LW, Kamping A (2005). No patrigenes required for femaleness in the haplodiploid wasp Nasonia vitripennis. Genetics (in press). Doi: 10.1534/genetics.105.044743.

Beye M (2004). The dice of fate: the csd gene and how its allelic composition regulates sexual development in the honey bee, Apis mellifera. BioEssays 26: 1131-1139.

Beye M, Hasselmann M, Fondrk MK, Page RE, Omold SW (2003). The gene csd is the primary signal for sexual development in the honeybee and encodes an SR-type protein. Cell 114: 419-429.

Bull JJ (1983). Evolution of Sex Determining Mechanisms. Benjamin/Cummings Publishing Company: Menlo Park, CA.

Caspari S (1958). An X-ray sperm-dose-action curve for mutations at a single locus in Mormoniella [ = Nasonia $]$. Radiat Res 8: 273-283.

Cook JM (1993). Sex determination in the Hymenoptera: a review of models and evidence. Heredity 71: 421-435.

Cook JM, Crozier RH (1995). Sex determination and population biology in the Hymenoptera. Trends Ecol Evol 10: 281-286.

Croteau S, Polychronakos C, Naumova A (2001). Imprinting defects in mouse embryos: stochastic errors or polymorphic phenotype? Genesis 31: 11-16.

DeChiara TM, Robertson EJ, Efstratiadis A (1991). Parental imprinting of the mouse insulin-like growth factor II gene. Cell 64: 849-859.

Dobson S, Tanouye M (1998). Evidence for a genomic imprinting sex determination mechanism in Nasonia vitripennis (Hymenoptera: Chalcidoidea). Genetics 149: 233-242.

Evans JD, Shearman DCA, Oldroyd BP (2004). Molecular basis of sex determination in haploidiploids. Trends Ecol Evol 19: 1-3.

Gadau J, Page RE, Werren JH (1999). Mapping of hybrid incompatibility loci in Nasonia. Genetics 153: 1731-1741.

Haig D (1998). Mother's boy or daddy's girl? Sex determination in Hymenoptera. Trends Ecol Evol 13: 380-381.

Herrick G, Seger J (1999). Imprinting and paternal genome elimination in insects. In: Henning W, Nover L, Scheer U (eds) Genomic Imprinting: An Interdisciplinary Approach. Results and Problems in Cell Differentiation, Vol, 25. SpringerVerlag: Berlin, pp 41-64.

Lloyd V (2000). Parental imprinting in Drosophila. Genetica 109: 35-44.

Muller HJ (1932). Further studies on the nature and causes of gene mutations. Proc Sixth Int Congr Genet 1: 213-255.
Page RE, Gadau J, Beye M (2002). The emergence of hymenopteran genetics. Genetics 160: 375-379.

Pultz M, Leaf D (2003). The jewel wasp Nasonia: querying the genome with haplo-diploid genetics. Genesis 35: 185-191.

Pultz MA, Zimmerman K, Alto NM, Kaeberlein M, Lange SK, Pitt JN et al (2000). A genetic screen for zygotic embryonic lethal mutations affecting cuticular morphology in the wasp Nasonia vitripennis. Genetics 154: 1213-1229.

Reik W, Walter J (2001). Genomic imprinting: parental influence on the genome. Nat Rev Genet 2: 21-32.

Ruetten K, Pietsch C, Olek K, Neusser M, Beukeboom LW, Gadau J (2004). Chromosomal anchoring of linkage groups and identification of wing size QTL using markers and FISH probes derived from microdissected chromosomes in Nasonia (Pteromalidae: Hymenoptera). Cytogenet Genome Res 105: 126-133.

Sakatani T, Wei M, Katoh M, Okita C, Wada D, Mitsuya K et al (2001). Epigenetic heterogeneity at imprinted loci in normal populations. Biochem Biophys Res Commun 283: 1124-1130.

Saul GB, Kayhart M (1956). Mutants and linkage in Mormoniella. Genetics 41: 930-937.

Saul GB, Saul SW, Becker S (1967). Linkage in Mormoniella. Genetics 57: 369-384.

Saul GB, Whiting PW, Saul SW, Heidner CA (1965). Wild-type and mutant stocks of Mormoniella. Genetics 52: 1317-1327.

Shuker D, Lynch J, Morais AP (2003). Moving from model to non-model organisms? Lessons from Nasonia wasps. BioEssays 25: 1247-1248.

Skinner S, Werren J (1980). The genetics of sex determination in Nasonia vitripennis. Genetics 94: S98.

Spencer H (2002). The correlation between relatives on the supposition of genomic imprinting. Genetics 161: 411-417.

Surani MA (2001). Reprogramming of genome function through epigenetic inheritance. Nature 414: 122-128.

Tram U, Sullivan W (2002). Role of delayed nuclear envelope breakdown and mitosis in Wolbachia-induced cytoplasmic incompatibility. Science 296: 1124-1226.

Werren JH (1997). Biology of Wolbachia. Ann. Rev. Entom. 42: 587-609.

Werren JH, Nur H, Eickbush D (1987). An extrachromosomal factor causing loss of paternal chromosomes. Nature 327: 75-76.

Whiting PW (1956). Spontaneous eye-color mutation in Mormoniella (the opening of Pandora's box). Proc Pa Acad Sci 30: 226-246.

Whiting PW (1960). Polyploidy in Mormoniella. Genetics 45: 949-970.

Whiting AR (1965). The complex locus $\mathrm{R}$ in Mormoniella vitripennis (Walker). Adv Genet 13: 341-358.

Whiting AR (1967). The biology of the parasitic wasp Mormoniella vitripennis [ = Nasonia brevicornis] (Walker). Quart Rev Biol 43: 333-406.

Wilkie SOM (1994). The molecular basis of genetic dominance. J Med Genet 31: 89-98.

Supplementary information accompanies the paper on the Heredity website (http://www.nature.com/hdy). 